\title{
Methodology for the abstraction and morphological experimentation of nature's patterns: an application case of morphology research subject in Industrial Design program
}

\author{
Elsie Arbeláez-Ochoa \\ Universidad Pontificia Bolivariana | Colombia | elsie.arbelaez@upb.edu.co \\ David A. Torreblanca-Díaz \\ Universidad Pontificia Bolivariana | Colombia | david.torreblanca@upb.edu.co \\ Karen Rodríguez-Castrillón \\ Universidad Pontificia Bolivariana | Colombia | karen.rodriguezc@upb.edu.co \\ Daniela Muñoz-Noriega \\ Universidad Pontificia Bolivariana | Colombia | daniela.munoz@upb.edu.co
}

\begin{abstract}
Forms in nature have been emerging in response to different requirements, in a complex and dynamic ecosystem. Architects and designers have usually used references from nature for their projects; in industrial design education, the use of nature's referents allows to expand the morphological possibilities for product design and systems. In this paper, a methodological proposal for the abstraction and morphological transformation of nature's patterns is presented, highlighting the advantages of parametric design and additive manufacturing technologies for morphological experimentation, in the context of the first application case of morphology research subject in the Industrial Design program at Universidad Pontificia Bolivariana.
\end{abstract}

Keywords: Bio-inspired design; Morphology; Experimental morphology; Additive manufacturing technologies; Industrial design education.

\section{INTRODUCCIÓN}

Las formas en la naturaleza son abundantes y diversas, han estado emergiendo por millones de años en respuesta a diferentes requerimientos, en un ecosistema complejo y en continua transformación, donde las diferentes especies de seres vivos y elementos inertes están interconectados. Stevens (1987) asevera que las formas existentes en la naturaleza son aquellas que, teniendo en cuenta las posibilidades tienen mayor probabilidad de existir, la naturaleza utiliza un número reducido de formas y patrones para diferentes fines y contextos; por ejemplo, predominan las formas curvas, los espirales, las formas serpenteantes, las ramificaciones y uniones en $120^{\circ}$, entre otras. La variedad morfológica se origina como resultado de la combinación de este limitado número de geometrías. Wagensberg (2005) plantea que las morfologías de la naturaleza están directamente relacionadas a una función: la esfera protege, el hexágono pavimenta, la espira empaqueta, la hélice agarra, el ángulo penetra, la onda comunica

Por otra parte, la experimentación morfológica es una herramienta utilizada ampliamente por los diseñadores para el desarrollo de proyectos con un enfoque de innovación disruptiva; esta experimentación, entendida como una de las posibilidades para proponer nuevos paradigmas morfológicos, ha sido poco documentada desde lo científico y lo académico por el gremio del diseño industrial (Sierra \& Patiño, 2005), y esto ha generado que los productos resultantes de ella se presenten más como piezas únicas de una alta novedad formal y complejidad, pero difícilmente serializables (Trujillo \& Valencia, 2012). La morfología experimental puede definirse entonces como el resultado de un proceso activo y vivencial de exploración geométrica, en el que se hace uso de herramientas de tipo experimental tanto físicas como digitales (Valencia, 2009; Trujillo \& Valencia, 2012).

Hoy en día, la experimentación morfológica es potenciada con las tecnologías digitales emergentes, tales como el diseño paramétrico-asociativo y generativo, las tecnologías de fabricación digital, la inteligencia artificial, la robótica, entre otros. Estas herramientas digitales permiten generar morfologías más complejas y adaptativas, trayendo consigo una nueva manera para enfrentar el proyecto y su representación. Dalla (2015) plantea que en el ámbito de la arquitectura, se ha integrado en las últimas décadas el diseño paramétrico al proyecto, como una nueva estrategia en el proceso de diseño, donde parámetros y componentes asociados crean un sistema matemático/algorítmico de generación geométrica dinámica que permite explorar más de un resultado como posible solución a un problema proyectual; las experiencias internacionales han evidenciado el uso de diseño paramétrico para la generación de componentes dinámicos (pieles responsivas, materiales reactivos), en 
estrategias de intervención urbanísticas (correlaciones complejas) o edilicias (articulación, organización) proponiendo nuevos paradigmas en el ámbito proyectual.

En este contexto en el cual irrumpen diferentes tecnologías digitales, se destaca la fabricación digital aditiva, comúnmente conocida como impresión 3D, una tecnología en auge y todo indica que su impacto en nuestra sociedad va a ser extraordinario porque supone un cambio radical para los modos de producción y distribución de productos; estas técnicas tienen ventajas sin precedentes, tales como la materialización de formas complejas, la fabricación de componentes mecánicos ya ensamblados, integración de diversos materiales o multi-materialidad, piezas únicas y personalizadas, con una amplia variedad de técnicas y materiales (Berchon y Luyt, 2016).

Las ventajas de las tecnologías digitales emergentes impulsan y enriquecen el desarrollo de proyectos de diseño que involucran experimentación morfológica, que hoy en día aumentan en forma incremental la complejidad geométrica de las propuestas. En este texto se presentan los primeros resultados de una propuesta metodológica para la abstracción y transformación morfológica experimental de patrones extraídos de la naturaleza, aprovechando su amplia variedad geométrica, destacando las ventajas del diseño paramétrico y la fabricación digital aditiva. Con este proyecto se espera obtener un método e insumos para ser usados en asignaturas de morfología, especialmente para ejercicios de experimentación morfológica bio-referenciada; estos resultados también podrían ser aplicados en procesos proyectuales en etapas de desarrollo formal, en asignaturas de taller de diseño. Se analizan dos casos realizados en la asignatura de investigación en morfología, programa de Diseño Industrial en la Universidad Pontificia Bolivariana, en Medellín, Colombia.

\section{METODOLOGÍA}

Se propone desarrollar un análisis exploratorio, a través de una metodología de tipo empírico-analítico, con énfasis en la experimentación. Los estudiantes de la asignatura de investigación en morfología del programa de diseño industrial complementan el uso de técnicas analógicas y digitales en el proceso de observación-abstracciónexperimentación-materialización. En figura 1 se puede ver la secuencia metodológica que empieza con la identificación y observación de patrones de la naturaleza, continúa con la síntesis morfológica, clasificación de patrones, transformación morfológica experimental a través de herramientas CAD (Computer Aided Design) y finaliza con la materialización de muestras a través de tecnologías de fabricación aditiva.

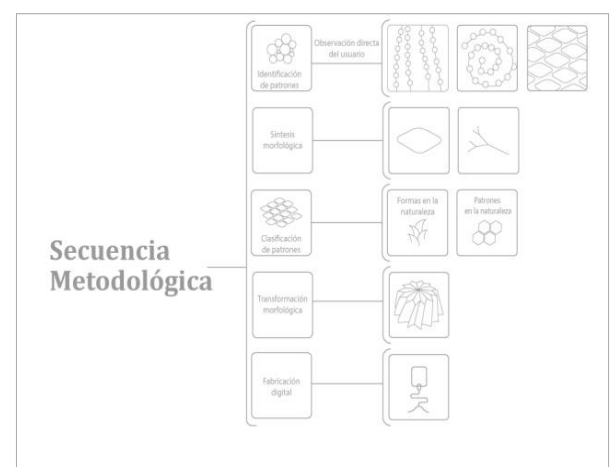

Figura 1:Secuencia metodológica propuesta por los autores, dibujo realizado por Daniela Muñoz Noriega.

A continuación, se describen las etapas de la propuesta metodológica.

\section{ETAPA 1: IDENTIFICACIÓN DE PATRONES}

Se inicia el proceso través una primera aproximación con el individuo natural o sistema, con el objeto de observar y analizar su morfología, comprender su forma general, superficies y texturas, así como su morfología local y patrones. Se define patrones como un conjunto elementos geométricos modulares que se repiten en el espacio configurado por una estructura visual reticular, un tipo de organización geométrica abundante en la naturaleza.

También es posible realizar diversos cortes transversales para entender la configuración morfológica interior y con esto profundizar la comprensión de la configuración geométrica del elemento o sistema natural. Se hace un registro fotográfico de los individuos observados.

\section{ETAPA 2: SÍNTESIS MORFOLÓGICA}

Una vez analizada la geometría global y local, su configuración exterior / interior e identificación de patrones, se hace una síntesis morfológica de los patrones encontrados. El proceso de abstracción geométrica se hace primero a través de bocetos analógicos, luego dibujos digitales en el software Illustrator@), posteriormente modelos digitales tridimensionales en el software CAD Rhinoceros@ y parametrizaciones con la herramienta Grasshopper(C).

\section{ETAPA 3: CLASIFICACIÓN DE PATRONES}

Después de la abstracción de patrones se hace una clasificación morfológica del individuo o individuos. La clasificación se realiza a partir del análisis de las formas predominantes en la naturaleza, según Arbeláez y Patiño (2009) y la clasificación morfológica patrones y texturas en la naturaleza propuesta por Patiño, E., Torreblanca-Díaz, D. A., Valencia-Escobar, A., \& Zuleta, A. (2019). Ver figuras 2 y 3 . 


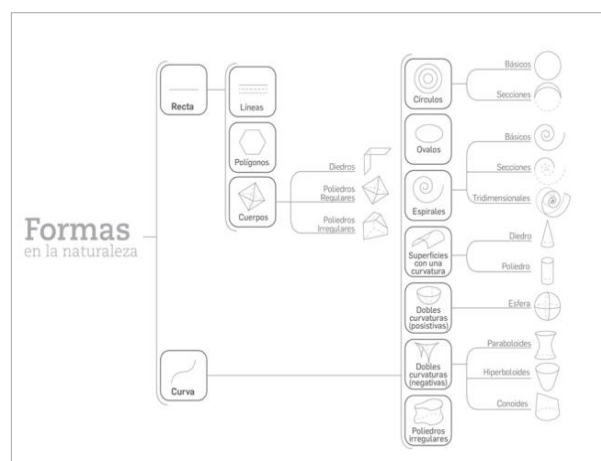

Figura 2: Clasificación de formas en la naturaleza, esquema hecho por los autores y basado en Arbeláez y Patiño (2009).

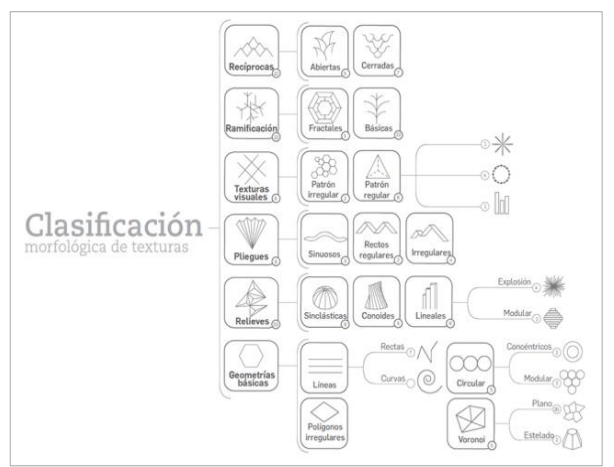

Figura 3: Clasificación morfológica de patrones y texturas, esquema hecho por los autores y basado en Patiño, E., Torreblanca-Díaz, D. A., Valencia-Escobar, A., \& Zuleta, A. (2019).

\section{ETAPA 4: TRANSFORMACIÓN MORFOLÓGICA}

\section{EXPERIMENTAL}

Una vez hecha la clasificación de los patrones estudiados, se propone hacer las transformaciones morfológicas experimentales y parametrizadas. Se pueden hacer de manera libre y experimental transformaciones geométricas tales como giros, rotaciones, reflejos, dilataciones, torsiones, entre otros.

\section{ETAPA 5: FABRICACIÓN DIGITAL ADITIVA}

Después de hacer las transformaciones morfológicas experimentales, se realiza la materialización de muestras a través de fabricación digital aditiva, aprovechando las amplias ventajas que tiene esta tecnología para representar morfologías de la naturaleza, tales como el alto nivel de precisión, fabricación de piezas complejas antes irrealizables con técnicas tradicionales, la multimaterialidad, entre otros. Se seleccionan los modelos digitales a imprimir, se revisan y preparan los archivos, se selecciona la tecnología de fabricación digital aditiva, el material y finalmente se materializan las piezas.

Según 3Dhubs (2020) las tecnologías de fabricación digital aditiva, popularmente conocidas como impresión 3D, se clasifican en categorías según el proceso utilizado: fotopolimerización, extrusión, granulado, inyección de aglutinante y fabricación de objetos laminados. La selección de la tecnología y el material responde a criterios técnicos y a otros contextuales, tales como las posibilidades de acceder a la tecnología y el presupuesto disponible.

\section{RESULTADOS}

Para este texto se seleccionaron dos casos desarrollados en la asignatura de investigación en morfología, programa de Diseño Industrial en la Universidad Pontificia Bolivariana, en Medellín, Colombia. Los casos elegidos fueron los trabajos hechos por las estudiantes Karen Rodríguez-Castrillón y Daniela Muñoz-Noriega, resultado del análisis de las especies Actinidia deliciosa, comúnmente conocida como Kiwi y Echinopsis Zucc, llamada popularmente como Cactus.

\section{CASO UNO: ANÁLISIS DE KIWI}

Se hizo una observación y análisis del individuo para comprender su forma general, superficies, texturas y patrones. También se realizaron cortes longitudinales y transversales del kiwi para entender la organización morfológica interior del fruto, en la figura 4 se pueden ver fotos del individuo analizado.

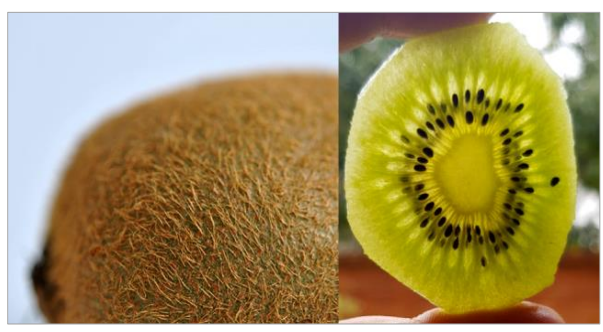

Figura 4: Especie Actinidia deliciosa. Fotografías de David A. Torreblanca-Díaz y la estudiante Daniela Muñoz Noriega.

Luego se hicieron bocetos analógicos, representando las formas iniciales de los módulos observados, posterior a un proceso de depuración geométrica se dibujan en el software Illustrator(C. Los patrones obtenidos se organizan considerando la clasificación de formas en la naturaleza según Arbeláez y Patiño (2009) y patrones y texturas en la naturaleza propuesta por Patiño, E.,

Torreblanca-Díaz, D. A., Valencia-Escobar, A., \& Zuleta, A. (2019). En la figura 5 se puede apreciar la abstracción bidimensional y clasificación morfológica de los módulos extraídos del Kiwi.

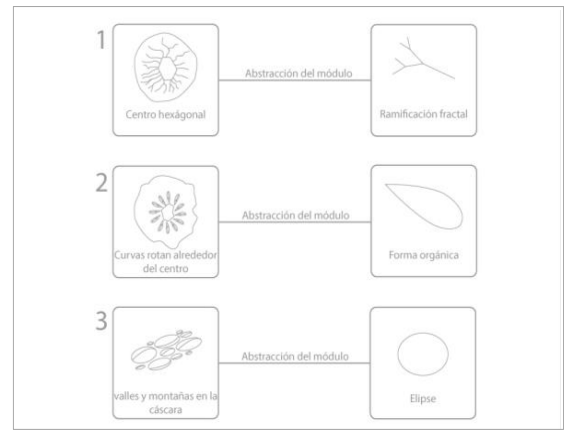

Figura 5: Abstracción morfológica bidimensional de módulos extraídos de la especie Actinidia deliciosa, 
esquema hecho por los autores.

Dibujo realizado por la estudiante Daniela Muñoz Noriega.

Posteriormente se hicieron transformaciones morfológicas experimentales bidimensionales (2D) y tridimensionales (3D). En la figura 6 se pueden ver las transformaciones bidimensionales, usando rotaciones y dilataciones.

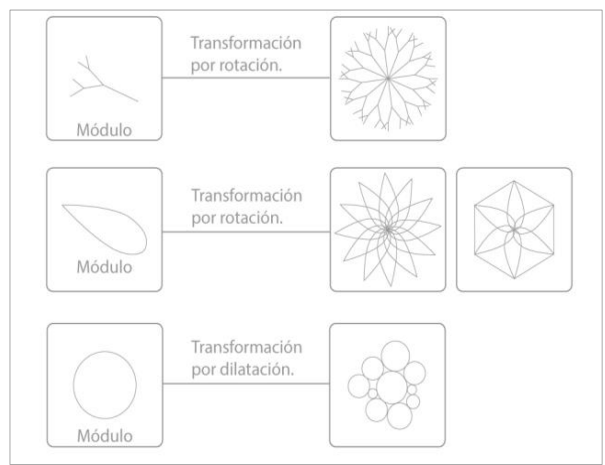

Figura 6: Trasformaciones bidimensionales (2D) en el software Illustrator@),

dibujos hechos por la estudiante Daniela Muñoz-Noriega.

Se realizaron diversas transformaciones morfológicas tridimensionales en el software CAD Rhinoceros@. En la figura 7 se puede apreciar la transformación hecha con el patrón fractal, se hizo una extrusión, se usaron las herramientas de rotación, Taper y Twist para girar la geometría con respecto su centro.

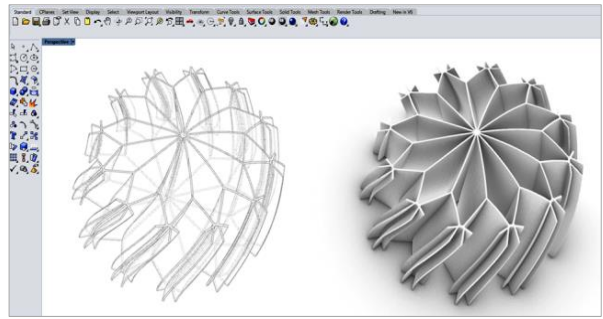

Figura 7: Transformaciones morfológicas experimentales en el software CAD Rhinoceros@ $\odot$. Modelos digitales realizados por la estudiante Daniela Muñoz Noriega.

Finalmente, se materializó la geometría obtenida como resultado de las transformaciones morfológicas tridimensionales del patrón fractal del Kiwi. La fabricación de la pieza se hizo a través de impresión 3D, se usa la máquina Anycubic Photon modelo S, tecnologia Digital Light Processing (DLP), esta impresora usa un rayo UV para solidificar las capas sucesivas de resina termosensible, se pueden obtener piezas de alta definición hasta de 47 micrómetros. En la figura 8 se puede ver la pieza obtenida.

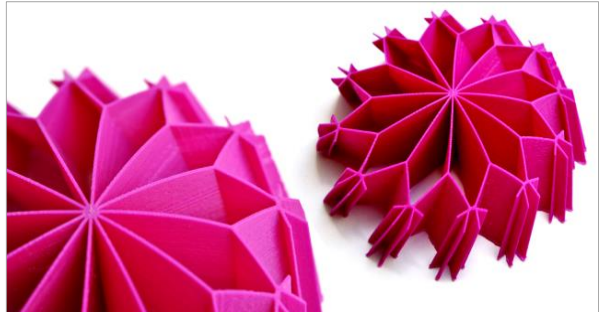

Figura 8: Materialización de una de las transformaciones hechas con el patrón fractal de la especie Actinidia deliciosa, a través de fabricación digital aditiva, máquina Anycubic Photon modelo S, tecnología Digital Light Processing (DLP).

Fotografías realizadas por David A. Torreblanca-Díaz.

\section{CASO DOS: ANÁLISIS DE CACTUS}

Tras un primer acercamiento a dos individuos pertenecientes a la especie Echinopsis Zucc, se reconoce la organización geométrica de la piel exterior y los módulos que se organizan en esa superficie. En la figura 9 se pueden ver individuo analizados.
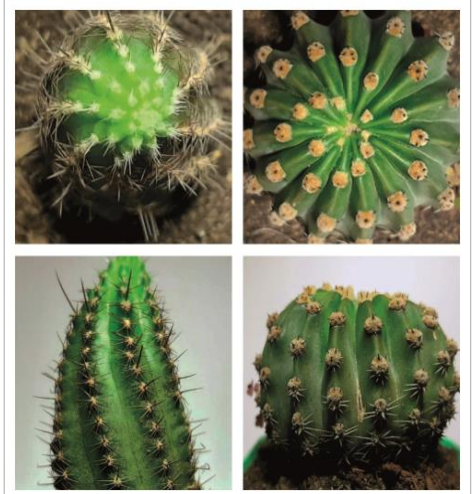

Figura 9: Individuos analizados pertenecientes a la especie Echinopsis Zucc, fotografías realizadas por la estudiante Karen Rodríguez-Castrillón.

Se hacen abstracciones bidimensionales en el software Illustrator@. Luego los módulos fueron clasificados según las formas en la naturaleza, propuesta de Arbeláez y Patiño (2009), ver figura 10.

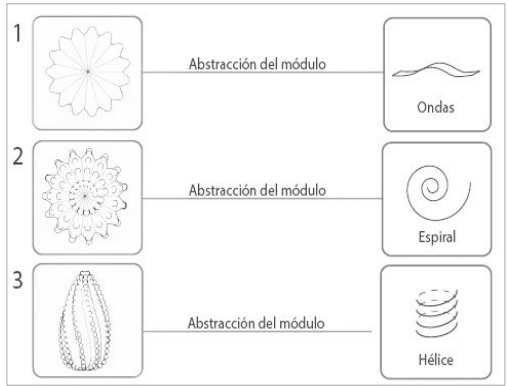


Figura 10: Abstracción morfológica bidimensional y clasificación de módulos extraídos de la especie Echinopsis Zucc. Esquema realizado por la estudiante Karen Rodríguez-Castrillón.

Mediante el software CAD (Computer Aided Design) Rhinoceros@ y la herramienta Grasshopper@, se generan abstracciones digitales paramétricas de los sistemas naturales observados, buscando representar contornos, simetrías, direcciones y movimientos. Ambos elementos estudiados tienen una morfología elipsoide, con superficies de doble curvatura estructuradas por nervaduras. El primer módulo observado dispone sus areolas creando una espiral esférica, el segundo módulo tiene hélices levógiras, terminando con un vórtice en su corona, ver figura 11.
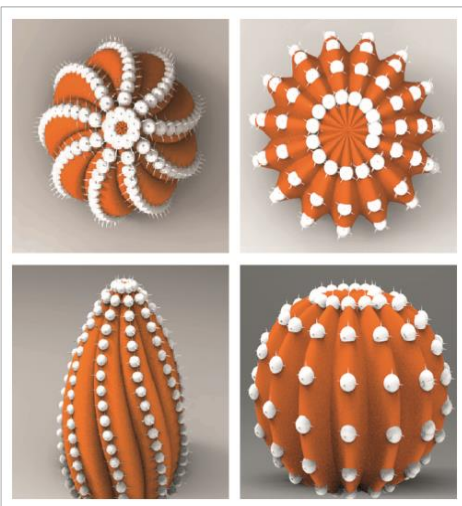

Figura 11: Abstracción digital tridimensional con el software CAD Rhinoceros@, individuos de la especie Echinopsis Zucc. Modelos digitales de la estudiante Karen Rodríguez-Castrillón.

A continuación, se generaron transformaciones morfológicas experimentales bidimensionales(2D) y tridimensionales (3D), usando como base las superficies curvas abstraídas del módulo del sistema natura observado. Con la herramienta Grasshopper@ del software CAD Rhinoceros@ los módulos se disponen de manera rotacional y traslacional en dirección dextrógira, se hace una rotación y desfase de curvas, extrusión, partición de sólido con esferoides y finalmente rotación de sólido sobre su propio eje. En la figura 12, 13 y 14 se pueden ver las transformaciones morfológicas experimentales, bidimensionales y tridimensionales.

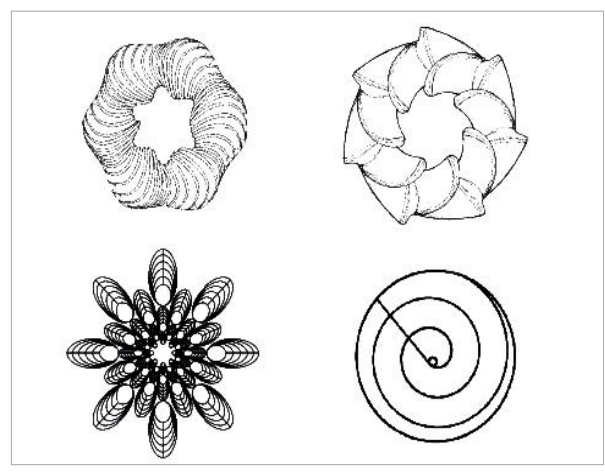

Figura 12: Trasformaciones bidimensionales experimentales de los individuos de la especie Echinopsis Zucc, en el software
Illustrator@. Esquema hecho por la estudiante Karen Rodríguez-Castrillón.

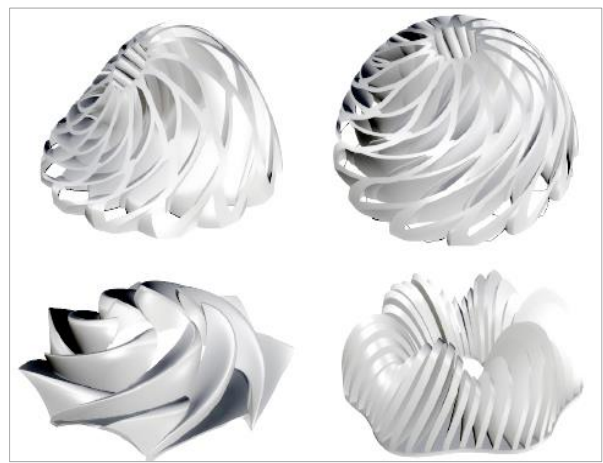

Figura 13: Transformaciones tridimensionales experimentales hechas con el software CAD Rhinoceros@, individuos de la especie Echinopsis Zucc. Modelos digitales realizados por la estudiante Karen Rodríguez-Castrillón.

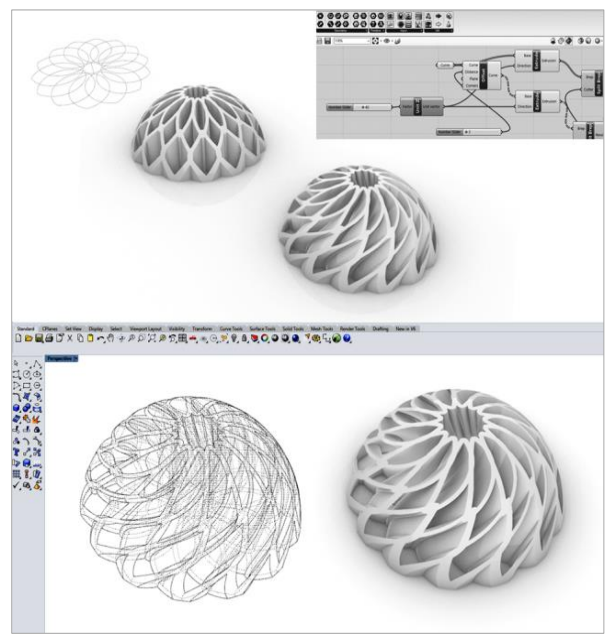

Figura 14: Transformaciones tridimensionales experimentales hechas con la herramienta Grasshopper( en el software CAD Rhinoceros@, individuos de la especie Echinopsis Zucc. Modelos digitales realizados por la estudiante Karen Rodríguez-Castrillón.

Finalmente, se materializó la geometría con las transformaciones de rotación dextrógira de curvaturas dobles sobre un eje central, a través de impresión 3D aditiva, máquina Anycubic Photon modelo $\mathrm{S}$, tecnología Digital Light Processing (DLP), ver figura 15.

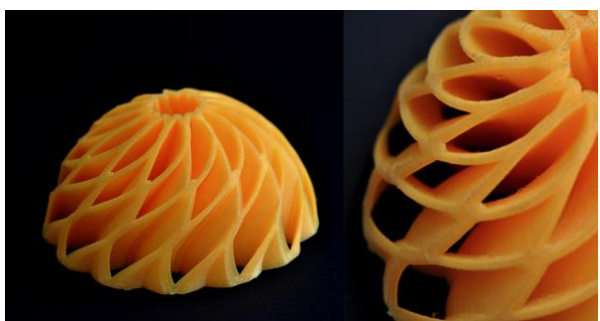

Figura 15: Materialización de una de las transformaciones experimentales de un módulo de la especie Echinopsis Zucc, a 
través de fabricación digital aditiva, máquina Anycubic Photon modelo S, tecnología Digital Light Processing (DLP). Fotografías

\section{DISCUSIÓN} de David A. Torreblanca-Díaz

Después de las actividades realizadas y la experiencia vivida con un enfoque exploratorio, en la asignatura de investigación en morfología, programa de Diseño Industrial en la Universidad Pontificia Bolivariana, emergen los siguientes análisis y conclusiones:

Se evidencia que la observación directa de los individuos naturales, a través de una primera aproximación multisensorial, es una herramienta efectiva para iniciar la caracterización morfológica e identificación de patrones, esto además es una experiencia atractiva y motivante para los estudiantes e investigadores. En la etapa de abstracción fue muy útil usar los principios de la geometría Euclidiana y la Gestalt, permitiendo definir las características geométricas de líneas, ángulos, superficies y usando la ley de la buena continuidad, de cierre, simetrías, entre otros. La etapa de clasificación morfológica se facilitó con el uso de la clasificación de formas en la naturaleza según Arbeláez y Patiño (2009) y patrones y texturas en la naturaleza propuesta por Patiño, E., Torreblanca-Díaz, D. A., Valencia-Escobar, A., \& Zuleta, A. (2019).

Se observan las ventajas de la integración de técnicas analógicas y digitales en el proceso de observaciónabstracción-experimentación-materialización, cumpliendo diferentes roles y sinergias en la trayectoria de actividades del proyecto. El registro a través de bocetos analógicos permite un rápido registro de los elementos naturales, aunque imprecisos permiten hacer una representación temprana e intuitiva para iniciar el proceso de abstracción morfológica, luego la representación bidimensional en el software Illustrator@ entrega una mayor precisión y facilitan la repetición de los módulos para el desarrollo de patrones bidimensionales, esto confluye en la realización de modelos digitales tridimensionales en el software CAD Rhinoceros@.

En esta primera experiencia, se validan las ventajas del diseño paramétrico-asociativo y la fabricación digital aditiva para la transformación morfológica experimental y su materialización. La parametrización con la herramienta Grasshopper@, da la posibilidad de generar modelos y transformaciones morfológicas dinámicas y adaptativas, esto facilita el proceso porque permite visualizar múltiples alternativas morfológicas en forma simultánea; gracias al uso de la tecnología de fabricación aditiva con la tecnología Digital Light Processing (DLP), fue posible materializar geometrías complejas, con un alto nivel de precisión y alta definición en su acabado superficial, sin requerir postprocesos y cumpliendo plenamente con el objetivo de representar transformaciones con piezas de apariencia.

Las limitaciones del proyecto tuvieron relación, por un lado, con restricciones técnicas; por ejemplo, algunos estudiantes no tenían el nivel necesario para las modelaciones digitales y no siempre se contaba con el computador adecuado para hacer modelaciones y parametrizaciones. Por otra parte, el curso de morfología tuvo pocos estudiantes y esto restringe la evaluación de los resultados. Por último, el proceso desarrollado se limitó solo a una signatura a lo largo de un semestre.

Una vez terminada esta primera etapa, aunque el alcance se limitó a fabricar las transformaciones morfológicas, se verifica la validez para utilizar patrones de la naturaleza como un referente geométrico porque es posible obtener una amplia variedad de formas, aprovechando las ventajas del diseño paramétrico-asociativo y la fabricación digital aditiva, en el contexto de ejercicios de morfología experimental.

Para el trabajo futuro es necesario aumentar la cantidad de validaciones de la metodología con estudiantes y con diferentes profesores para recibir una retroalimentación y hacer ajustes en la metodología. Finalmente, no se hizo un análisis sobre la posibilidad para usar las morfologías bioinspiradas obtenidas en procesos proyectuales, se espera a futuro incluir una nueva etapa para usar esas geometrías en proyectos de diseño a nivel académico y/o profesional.

\section{AGRADECIMENTOS}

Damos las gracias al profesor Santiago Diez Restrepo por todo el acompañamiento en el proceso de modelación digital, al técnico del laboratorio Yeison Alexander Gallego Ríos por facilitar las experimentaciones y fabricación de muestras. Así mismo, agradecemos a la Facultad de Diseño Industrial de la Universidad Pontificia Bolivariana por dar todas las facilidades para el desarrollo del proyecto.

\section{REFERENCIAS}

Berchon, Mathilde, y Luyt, Bertier. (2016). La impresión 3D: guía definitiva para makers, diseñadores, estudiantes, profesionales, artistas y manitas en general. Barcelona, ESPAÑA: Editorial Gustavo Gili, 2016. Pro Quest ebrary. Web. 29 September 2016

Dalla Costa, M. (2005). Sistemas Generativos Dinámicos. ARQUISUR REVISTA, 20-48.

Patiño, E., Arbeláez, E. (2009). Generación y transformación de la forma. Editorial Universidad Pontificia Bolivariana: Medellín.

Patiño, E., Torreblanca-Díaz, D. A., Valencia-Escobar, A., \& Zuleta, A. (2019). Classification, characterization, and abstraction of vegetable surfaces for design. International Journal of Design \& Nature and Ecodynamics, 14(2), 131-146.

Sierra, F., \& Patiño, E. (2005). Objetos a partir de la rigidización de estructuras de membrana. Iconofacto, 1(2).

Stevens, P. (1987) Patrones y pautas en la naturaleza. Salvat Editores: Barcelona, 1987.

Valencia-Escobar, A. (2009). Morfología experimental: esquema de formación en diseño industrial. En Ferrero L. (Ed), Libro de resúmenes Forma \& Contexto VII Congreso Nacional y IV Internacional. (pp. 82). Buenos Aires: Sema Ars Noa.

Wagensberg, J. (2005). La rebelión de las formas. Barcelona: Editorial Matemas.

3Dhubs. (2020). Additive manufacturing technologies: An overview. Recuperado de https://www.3dhubs.com/knowledgebase/additive-manufacturing-technologies-overview 